\title{
BILGE DUMP AUTOMATIC ALERT SYSTEM IN SOUTHERN AFRICA OCEANS
}

\author{
L.W. Mdakane ${ }^{1}$, R.G.V. Meyer ${ }^{1}$, B. Sibolla ${ }^{1, *}$ \\ 1 Spatial Information Systems, e-Government \\ Next Gen Enterprises and Institutions, CSIR \\ Pretoria, South Africa.
}

KEY WORDS: Bilge Waste Dumping, Oil Spill, Oceans, Image Classification, Synthetic Aperture Radar.

\begin{abstract}
:
Oil spill over the sea surface formed because of oil-tanker accidents or illegal bilge dumping of tankers can cause significant environmental damage depending on the location and amount. The international legislation contains minor and well-defined exceptions related to ocean areas (internal waters, marine protected areas, MARPOL "special" areas, territorial seas or exclusive economic zones). These areas often determine whether or not an action is considered legal/illegal and define the rights and obligations, including law enforcement obligations. Deliberated oil spill are often caused by vessels illegally discharging oily waste during cleaning operations. To minimise the ecological impact caused by the oil spill, a rapid response from the authorities is required. To facilitate the quick response, we propose an automated bilge dump alert system based on space-borne SAR analysis over Southern Africa oceans. The proposed alert system detects potential bilge dumps and classifies them according to confidence and alert levels. The confidence levels described the quality of the detected bilge dump, based on the probability measures of the observed bilge candidates. The alert levels described the enormity of the alert based on the detection location and confidence level. The system showed promising results in classifying bilge dumps according to the alert level.
\end{abstract}

\section{INTRODUCTION}

The lack of regular surveillance and the rapid growth of maritime transportation increases the threat of illegal bilge dumping (Xiong et al., 2015). The capability of Synthetic Aperture Radar (SAR) for long-term monitoring of operational oil pollution over the sea surface is well known (Solberg, 2012, Sankaran, 2019). SAR is an essential part of bilge dumping response because it offers several advantages such as a wide area, costeffective, most weather conditions, day and night surveillance at open sea. The approach is to detect the presence of bilge dumping from Synthetic Aperture Radar (SAR) imagery, including the ship that may have illegally dumped the bilge waste. In a SAR image, an oil slick appears as a darker feature (in contrast to the brighter sea surface) that is linearly shaped due to the movement of the dumping ship (Mdakane, Kleynhans, 2017). However, not only bilge dumps have these characteristics. Other naturally occurring phenomena can have similar features to bilge dump events known as look-alikes.

Despite the limitations, trained operators can visually detect oil spill discharges from vessels by analysing the spill candidate shape, the ocean conditions and the proximity of the vessel responsible (Solberg et al., 2007). However, a visual/manual based alert system of potential bilge dump at sea can be a time consuming, labour intensive and a subjective task when there are many SAR scenes to be checked (Alpers, Espedal, 2004). A more reliable bilge dumping alert system can be achieved using image processing techniques to automate the process (Mdakane, Kleynhans, 2017, Cantorna et al., 2019). The automated detection process can be broadly divided into three steps, darkspots detection, feature extraction and discrimination (Fingas, Brown, 2018). The systems are designed to detect oil slicks through a SAR image without the intervention of the expert. They identify and isolate all darker regions (potential bilge dumping events) present in the image. Features for each detection are

\footnotetext{
* Corresponding author
}

extracted and used to discriminate potential bilge dumps from look-alikes (Ferraro et al., 2010, Konik, Bradtke, 2016, Alpers et al., 2017). This paper focuses on the post-discrimination processing step, where possible bilge dump events have been identified and decisions have to be made.

Keramitsoglous et al. successfully demonstrated a fully automated system based on fuzzy logic (Keramitsoglou et al., 2006). The detections (potential bilge dump events) were assigned various confidence levels that indicated the quality of each detection. The confidence levels were based on a probability measure calculated using the detected dark regions and the extracted features (e.g., shape, size, texture, backscatter) (Brekke, Solberg, 2008). The authors presented detected oil slicks events with probability values, location, and oil slick attributes necessary for further analysis for end users (e.g., distance to land, size and shape). However, using SAR features alone may not be sufficient, and the system may require additional features for an improved confidence estimation. Additional data from SAR and other remote sensing data or through in-situ measurements can be used to retrieve wind information, the source of pollution source, known look-alikes, thus improving the confidence level associated with the SAR-based oil slick detection (Topouzelis et al., 2015).

In this paper, we present an automated bilge dump alert system based on SAR analysis over Southern Africa oceans to provide decision support to the user. The alert system detects potential bilge dump events and classifies them according to confidence and alert levels. The confidence levels described the quality of the detected bilge dump using probability measurements of the observed bilge candidates. The alert levels describe the enormity and risk of the the detected bilge dump based on the environmental sensitivity of detection location and confidence level. The paper is divided into five sections including the introduction, data description, methodology, results with discussion, and conclusions. 

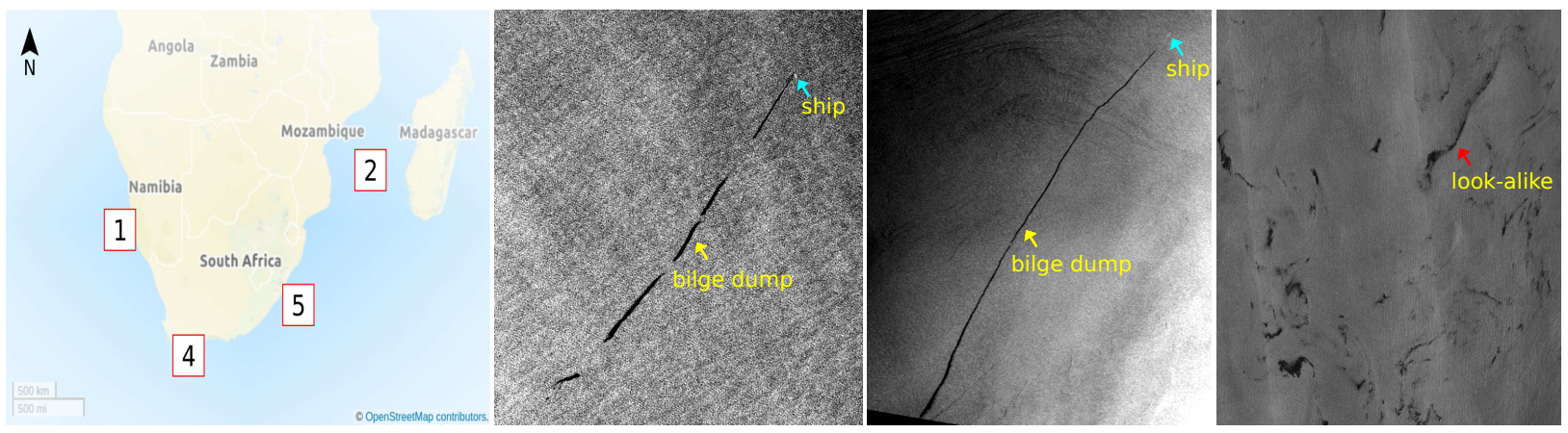

Figure 1. From Left to Right: 1) A Southern Africa region map showing the number of images and locations for the study. 2) Bilge dump between Mozambique and Juan de Nova Island coastal area (28 August 2015 Sentinel-1A IW mode). 3) Bilge dump over South Africa coastal area (14 April 2016 Sentinel-1A IW mode). 4) An example of Sentinel-1A (IW mode) with linear dark-spots that are not real bilge dumps (i.e., look-alikes).

\section{DATA DESCRIPTION}

There is a lack of studies for automated ocean monitoring applications in the Southern African coastal areas. However, the increasing availability of SAR satellite data can solve this problem (Torres et al., 2012). The study objective is to monitor oil pollution in these oceans using data that will be available on a regular basis. The study area covers the oceans surrounding three Africa countries, including Namibia, South Africa and Mozambique coastal areas, see map locations in figure (1). The data used in the study consisted of Sentinel-1 Ground Range, Multi-looked, Data (GRD) using two modes:

- Interferometric Wide Swath (IW) with $250 \mathrm{~km}$ swath, high $(10 \times 10 \mathrm{~m})$ and medium $(40 \times 40 \mathrm{~m})$ spatial resolutions.

- Extra-Wide Swath (EW) with $400 \mathrm{~km}$ swath, high (25 x 25 $\mathrm{m})$ and medium $(40 \times 40 \mathrm{~m})$ spatial resolutions.

Dual-polarisation products are obtained by operating the radar with one ( $\mathrm{H}$ or $\mathrm{V})$ polarisation on transmit and both simultaneously on receive. Sentinel-1 GRD modes have dual-polarisation, that is, co-polarisation (VV or $\mathrm{HH}$ ) and cross-polarisation $(\mathrm{VH}$ or HV). We only used co-polarisation images for the study due to the stronger backscattering properties in oil spill detection studies (Staples, Rodrigues, 2013, Fingas, Brown, 2014). Copolarisation products are obtained by operating the radar with the same $(\mathrm{H}$ or $\mathrm{V})$ polarisation on both transmit and receive.

\section{METHODOLOGY}

SAR discrimination is based mainly on experience and prior information on weather conditions, the difference in shape, the contrast to surrounding and background objects and proximity to land. The automated alert system resembled the expert's decisions and based on several guidelines, knowledge and rules.

The system comprises of multiple processes, including, input data enhancement, potential bilge dump segmentation, the classification with confidence levels and alert level reports, see flowchart in figure (2).

\subsection{Bilge feature database}

A SAR image is read and enhanced by masking out land pixels (using world land shape file), calibrated to sigma nought values and reduced noise with Lee speckle filter (Lee, 1981). The

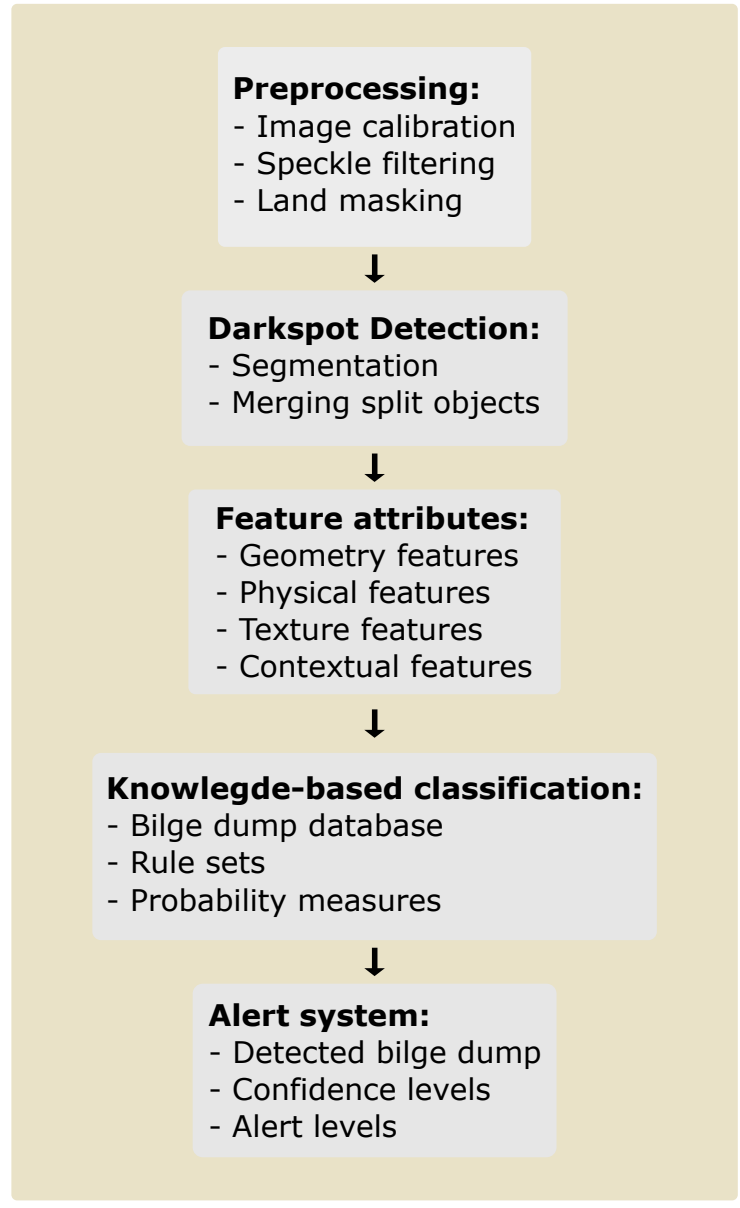

Figure 2. Bilge dump event alert system flowchart.

appropriate dark regions (potential bilge dumps) were detected using segmentation method described in (Mdakane, Kleynhans, 2017). The method uses an iterative dark-spot segmentation method while extracting potential bilge dumps (linear darkspots), see illustration in figure (3).

We extracted geometric features (length, width and the ratio of the length to width, denoted as aspect ratio), physical features (intensity mean, minimum, maximum, and standard deviation), and texture features (Haralick features) from the detected dark spots. High confidence bilge dump events were used to create a database of extracted features from visually detection bilge 


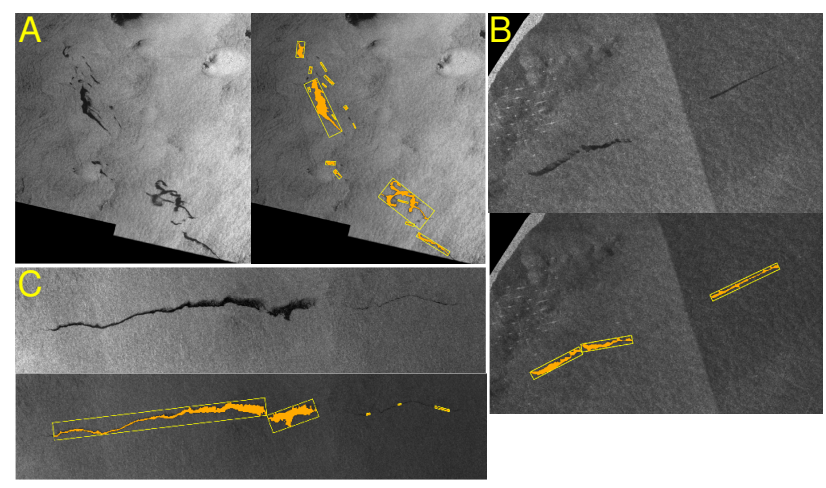

Figure 3. An example of detected potential bilge dump events (shown inside rectangles).

dumps, see example features in table (1).

\begin{tabular}{lcccc}
\hline & MIN & MAX & MEAN & STD DEV \\
\hline intensity value & 2.26 & 51.63 & 18.05 & 16.30 \\
length $(\mathrm{km})$ & 1.71 & 90.68 & 10.30 & 12.85 \\
width $(\mathrm{km})$ & 0.17 & 10.32 & 1.52 & 1.63 \\
length to width ratio & 1.72 & 38.91 & 7.58 & 5.75 \\
Area $\left(\mathrm{km}^{2}\right)$ & 0.12 & 125.99 & 7.70 & 17.02 \\
Object mean $(\mathrm{dB})$ & -29.31 & -16.50 & -23.48 & 2.64 \\
Background mean $(\mathrm{dB})$ & -23.51 & -12.75 & -19.13 & 2.62 \\
Mean ratio & 1.05 & 1.63 & 1.24 & 0.11 \\
\hline
\end{tabular}

Table 1. Bilge dump features database example.

\subsection{Confidence estimation}

The probability of each potential bilge dump, detected dark regions, was estimated using a rule base modelling system. The confidence levels estimated the likelihood of a dark region to be a bilge dump events as either high, medium or low. We determined the confidence levels based manual and automated analyses based on several guidelines. These were made up of a set of conditions based on geometric, physical and texture features were used to classify bilge waste from look-alikes. The guidelines used to estimate the confidence levels are presented in table (2). An example of a knowledge-based classification:

High Confidence: IF the wind level is moderate AND the length to width ratio is high AND the is a few neighbouring detections THEN confidence is HIGH.

The feature limits were automatically estimated using the bilge database in table (1).

\begin{tabular}{lccc}
\hline Feature & \multicolumn{3}{c}{ Confidence level } \\
values & Low & Medium & High \\
\hline wind speed & low & moderate & high \\
Number of detections & many & some & few \\
homogeneity & less & moderate & high \\
contrast & low & moderate & high \\
distance to ship & away & further & close \\
length to width ratio & low & medium & high \\
area & large or small & medium & large \\
\hline
\end{tabular}

Table 2. Feature guideline as observed manually and through literature

\subsection{Alerts estimation}

We only measured dark regions classified as oil spills for the alert level. The alerts, adopted from (Ferraro et al., 2010), were divided into red alert, alert, informative alert.

Red alert rule: IF ship/platform is close AND is close to shipping lane THEN red alert.

Alert rule: IF ship/platform is further AND is further to the shipping lane.

Informative alert rule: IF no/away ship AND away from shipping lane.

An expert visually obtained the ship/platform and shipping lane features were manually.

\section{RESULTS AND DISCUSSION}

The system was developed using twelve SAR images with 29 bilge dump events acquired over Southern Africa oceans. The confidence levels of each dark region were calculated based on the extracted features and classified as a potential bilge dump or look-alike. Potential bilge dumps were processed further to determine their alert levels based on location, ship information and confidence value. The output images and tables provide the user with all relevant information for supporting decisionmaking, these include, the confidence level of the detection and the enormity of the alert.

\begin{tabular}{lccc}
\hline Dark Region ID & {$\left[\right.$ Length $(\mathrm{km})$, Area $\left.\left(\mathrm{km}^{2}\right)\right]$} & Confidence & Alert type \\
\hline EWH.7A8B & {$[12.0,6.6]$} & high & red alert \\
IWH.9FD9 & {$[6.3,29]$} & high & red alert \\
IWH.OF14 & {$[4.6,14.8]$} & medium & informative \\
EWM.7B41 & {$[8.74,1.6]$} & medium & alert \\
IWH.E284 & {$[4.7,2.4]$} & low & informative \\
IWH.65DD & {$[6.7,4.7]$} & medium & informative \\
IWH.8137 & {$[6.1,3.3]$} & high & alert \\
IWM.C1CF & {$[12.8,3.0]$} & high & red alert \\
IWH.3B41 & {$[19.9,76.8]$} & low & informative \\
\hline
\end{tabular}

Table 3. Bilge dump confidence and alert system results table.

The system was compared with manual confidence and alert measures. The automated feature extraction results showed an overall performance of $93 \%$ for bilge dump detection. The proposed alert system output (see, Table (3)) showed an overall performance of $82 \%$ and $76 \%$, for confidence and alert level estimations, respectively. The system, however, had a small dataset, and performance can be improved by increasing the dataset that includes more African oceans.

\section{CONCLUSION}

We proposed an automated bilge dump alert system based on SAR analysis over Southern Africa oceans. The alert system detected potential bilge dumps and classifies them according to confidence and alert levels. The confidence levels described the quality of the detection, while the alert levels described the enormity of the of the bilge dump. We compared the system with manual confidence and alert measurements where it showed promising results. The bilge dump alert system provided information that allows the end user to make a decision on the 
follow-up action to be taken regarding the specific detection. Future work will test more SAR data by expanding the study area, incorporate information about ship traffic using density maps and the position of environmentally sensitive areas (e.g., marine protected areas). It will also include an automated ship or platform and ship lane feature extraction and a comparison with the state of art algorithms.

\section{REFERENCES}

Alpers, W., Espedal, H. A., 2004. Oils and surfactants. SAR marine user's manual, 263-275.

Alpers, W., Holt, B., Zeng, K., 2017. Oil spill detection by imaging radars: Challenges and pitfalls. Remote Sensing of Environment, 201, 133-147.

Brekke, C., Solberg, A. H. S., 2008. Classifiers and confidence estimation for oil spill detection in ENVISAT ASAR images. IEEE Geoscience and Remote Sensing Letters, 5(1), 65-69.

Cantorna, D., Dafonte, C., Iglesias, A., Arcay, B., 2019. Oil spill segmentation in SAR images using convolutional neural networks. A comparative analysis with clustering and logistic regression algorithms. Applied Soft Computing, 84, 105716.

Ferraro, G., Baschek, B., de Montpellier, G., Njoten, O., Perkovic, M., Vespe, M., 2010. On the SAR derived alert in the detection of oil spills according to the analysis of the EGEMP. Marine pollution bulletin, 60(1), 91-102.

Fingas, M., Brown, C., 2014. Review of oil spill remote sensing. Marine pollution bulletin, 83(1), 9-23.

Fingas, M., Brown, C. E., 2018. A review of oil spill remote sensing. Sensors, 18(1), 91.

Keramitsoglou, I., Cartalis, C., Kiranoudis, C. T., 2006. Automatic identification of oil spills on satellite images. Environmental modelling \& software, 21(5), 640-652.

Konik, M., Bradtke, K., 2016. Object-oriented approach to oil spill detection using ENVISAT ASAR images. ISPRS Journal of Photogrammetry and Remote Sensing, 118, 37-52.

Lee, J.-S., 1981. Refined filtering of image noise using local statistics. Computer graphics and image processing, 15(4), 380-389.

Mdakane, L. W., Kleynhans, W., 2017. An ImageSegmentation-Based Framework to Detect Oil Slicks From Moving Vessels in the Southern African Oceans Using SAR Imagery. IEEE Journal of Selected Topics in Applied Earth Observations and Remote Sensing.

Sankaran, K., 2019. Protecting oceans from illicit oil spills: environment control and remote sensing using spaceborne imaging radars. Journal of Electromagnetic Waves and Applications, 33(18), 2373-2403.

Solberg, A. H. S., 2012. Remote sensing of ocean oil-spill pollution. Proceedings of the IEEE, 100(10), 2931-2945.

Solberg, A. H. S., Brekke, C., Husoy, P. O., 2007. Oil spill detection in Radarsat and Envisat SAR images. IEEE Trans. Geosci. Remote Sens., 45(3), 746-755.
Staples, G., Rodrigues, D. F., 2013. Maritime environmental surveillance with radarsat-2. XVI Brazilian Remote Sens. Symp. (SBSR), INPE Foz do Iguaçu, Brazil, 8445-8452.

Topouzelis, K., Tarchi, D., Vespe, M., Posada, M., Muellenhoff, O., Ferraro, G., 2015. Detection, tracking, and remote sensing. Handbook of Oil Spill Science and Technology, 357-383.

Torres, R., Snoeij, P., Geudtner, D., Bibby, D., Davidson, M., Attema, E., Potin, P., Rommen, B., Floury, N., Brown, M. et al., 2012. GMES Sentinel-1 mission. Remote Sensing of Environment, 120, 9-24.

Xiong, S., Long, H., Tang, G., Wan, J., Li, H., 2015. The management in response to marine oil spill from ships in China: A systematic review. Marine pollution bulletin, 96(1-2), 7-17. 